\title{
Late preterm prelabor rupture of fetal membranes: fetal inflammatory response and neonatal outcome
}

\author{
Ivana Musilova ${ }^{1}$, Ctirad Andrys ${ }^{1}$, Marcela Drahosova ${ }^{1}$, Barbora Zednikova ${ }^{1}$, Helena Hornychova ${ }^{2}$, Lenka Pliskova ${ }^{3}$,
} Helena Zemlickova ${ }^{4}$, Bo Jacobsson ${ }^{5}$ and Marian Kacerovsky ${ }^{6}$

BACKGROUND: To characterize the influence of microbial invasion of the amniotic cavity (MIAC) and/or intra-amniotic inflammation (IAI) on the intensity of the fetal inflammatory response and the association between the presence of the fetal inflammatory response syndrome (FIRS) and short-term neonatal morbidity in the preterm prelabor rupture of membranes (PPROM) between the gestational ages of 34 and 37 weeks.

METHODS: One hundred and fifty-nine women were included in the study. The umbilical cord blood interleukin (IL)-6 concentrations were determined using enzyme-linked immunosorbent assay kits. FIRS was defined based on the umbilical cord blood IL-6 concentration and the presence of funisitis and/or chorionic plate vasculitis.

RESULTS: Women with both MIAC and IAI had the highest median umbilical cord blood IL-6 concentrations and highest rates of FIRS. Women with FIRS had the higher rates of earlyonset sepsis and intraventricular hemorrhage grades I and II when FIRS was characterized based on the umbilical cord blood IL-6 concentrations and the histopathological findings. CONCLUSION: The presence of both MIAC and IAl was associated with a higher fetal inflammatory response and a higher rate of FIRS. Different aspects of short-term neonatal morbidity were related to FIRS when defined by umbilical cord blood IL-6 concentrations and the histopathology of the placenta.

$\mathbf{P}$ reterm prelabor rupture of membranes (PPROM), responsible for about one-third of all preterm deliveries, is characterized by the rupture of fetal membranes and leaking of amniotic fluid before the onset of regular uterine activity before the gestational age of 37 weeks (1-3). In general, PPROM presents in $2-8 \%$ of all pregnancies (1-3).

PPROM pregnancies are often complicated by the presence of hostile intra-amniotic conditions such as microbial invasion of the amniotic cavity (MIAC) and intra-amniotic inflammation (IAI) (4-6). These conditions usually lead to the development of acute histological chorioamnionitis (HCA) followed by the activation of the fetal innate immune system (fetal inflammatory response), characterized by an elevation of inflammatory mediators in fetal blood (5,7-9). This situation results in multisystem involvement and the development of the fetal inflammatory response syndrome (FIRS), which might progress toward multiorgan dysfunction and failure (8-11).

On the basis of the gestational age at the occurrence of the rupture of the fetal membranes, PPROM can be subdivided into the following two subgroups: PPROM occurring before 34 weeks of gestation and PPROM occurring between 34 and 37 weeks of gestation $(1,3)$. The latter represents a more frequent form of PPROM, which is characterized by a very low incidence of serious short-term neonatal morbidity, and might result in long-term sequelae, such as necrotizing enterocolitis, bronchopulmonary dysplasia, and severe intraventricular hemorrhage grades (12-15). On the other hand, PPROM between 34 and 37 weeks of gestation is still complicated by a relatively higher incidence of respiratory morbidity and the risk of early-onset sepsis (12-15).

In spite of the fact that the occurrence of intra-amniotic complications decreases with advanced gestational age, approximately every fifth and tenth case of PPROM between 34 and 37 weeks is complicated by MIAC and IAI, respectively $(4,12)$. The presence of MIAC and acute HCA in PPROM between 34 and 37 weeks has been shown to be associated with the highest intensity of the intra-amniotic inflammatory response, measured by interleukin-6 (IL-6) concentrations in amniotic fluid, and a higher rate of earlyonset sepsis and severe neonatal morbidity (12). Nevertheless, in contrast with PPROM before 34 weeks, there is a paucity of information about the intensity of the fetal inflammatory response and the presence of FIRS in terms of the intraamniotic environment, characterized by MIAC and/or IAI, in

\footnotetext{
${ }^{1}$ Department of Clinical Immunology and Allergy, University Hospital in Hradec Kralove, Charles University, Faculty of Medicine Hradec Kralove, Hradec Kralove, Czech Republic; ${ }^{2}$ Fingerland's Department of Pathology, University Hospital in Hradec Kralove, Charles University, Faculty of Medicine Hradec Kralove, Hradec Kralove, Czech Republic; ${ }^{3}$ Institute of Clinical Biochemistry and Diagnostics, University Hospital in Hradec Kralove, Charles University, Faculty of Medicine Hradec Kralove, Hradec Kralove, Czech Republic; ${ }^{4}$ Institute of Clinical Microbiology, University Hospital in Hradec Kralove, Charles University, Faculty of Medicine Hradec Kralove, Hradec Kralove, Czech Republic; ${ }^{5}$ Department of Obstetrics and Gynecology, Sahlgrenska Academy, Gothenburg University, Gothenburg, Sweden; ${ }^{6}$ Department of Obstetrics and Gynecology, University Hospital in Hradec Kralove, Charles University, Faculty of Medicine Hradec Kralove, Hradec Kralove, Czech Republic. Correspondence: Marian Kacerovsky (kacermar@fnhk.cz) 
pregnancies complicated by PPROM between 34 and 37 weeks of gestation.

Therefore, the main aim of the present study was to characterize the influence of MIAC and/or IAI on the intensity of the fetal inflammatory response in PPROM between gestational ages 34 and 37 weeks. The second aim was to investigate the association between the presence of FIRS, defined based on the umbilical cord blood IL-6 concentrations and based on the histopathology of the placenta, and short-term neonatal morbidity in PPROM between gestational ages 34 and 37 weeks. The last objective of this study was to determine the potential association between the gestational age at delivery in PPROM between gestational ages 34 and 37 weeks and short-term neonatal morbidity.

\section{METHODS}

\section{Patient Population}

A prospective cohort study of pregnant women with PPROM between gestational ages $34+0$ and $36+6$, who were admitted to the Department of Obstetrics and Gynecology, University Hospital Hradec Kralove between August 2014 and May 2017, was conducted. Women with singleton pregnancies who were at least 18 years old were included in the study. Women with diabetes mellitus, gestational diabetes mellitus, preeclampsia, hypertension-either chronic or pregnancy-induced-the presence of chromosomal or structural fetal abnormalities, signs of hypoxia of the fetus, or significant vaginal bleeding were excluded from the study. During the study period, 264 women with singleton pregnancies complicated by PPROM between the gestational ages of $34+0$ and $36+6$ weeks were admitted. In total, 204 women provided their written consent. The amniotic fluid, umbilical cord blood, and placental results were available for 171 women.

Gestational ages were established by first-trimester fetal biometry. Women with gestational ages between $34+0$ and $34+6$ weeks were treated with both corticosteroids and antibiotics, whereas only antibiotics were administered to those beyond gestational age 34 +6 weeks. Women with proven bacteria in their amniotic fluid and/ or point-of-care amniotic fluid IL-6 concentration of $\geq 745 \mathrm{pg} / \mathrm{ml}$ were managed actively. The remaining women were managed conservatively. PPROM was diagnosed by examination with a sterile speculum to verify the pooling of amniotic fluid in the vagina. In situations of clinical doubt, amniotic fluid leakage was confirmed by the presence of insulin-like growth factor-binding proteins (ACTIM PROM test; MedixBiochemica, Kauniainen, Finland) in the vaginal fluid.

\section{Sample Collection}

Ultrasound-guided transabdominal amniocentesis was performed upon admission before the administration of corticosteroids and antibiotics; 1-2 ml of amniotic fluid was obtained. A total of $100 \mu \mathrm{l}$ of non-centrifuged amniotic fluid was used for the bedside assessment of IL-6 concentrations. Two aliquots of non-centrifuged amniotic fluid were immediately transported to the microbiology laboratory for PCR testing for Ureaplasma species, Mycoplasma hominis, Chlamydia trachomatis, and the 16S rRNA gene, as well as for aerobic/anaerobic cultivation of amniotic fluid.

Umbilical blood samples were obtained by venipuncture from clamped umbilical cords immediately after the delivery of the neonates and before the delivery of the placenta using a vacutainer blood-collecting system. The samples of umbilical cord blood were immediately centrifuged and aliquoted, and the supernatants were stored at $-70^{\circ} \mathrm{C}$ until assayed.

At delivery, the placenta, the fetal membranes, and the umbilical cord were fixed in 10\% neutral buffered formalin. Tissue samples were obtained from the placenta (at least two samples), umbilical cord (usually one sample), and fetal membranes (at least two samples), and then routinely processed and embedded in paraffin. Sections of tissue blocks were stained with hematoxylin and eosin.

The study was approved by the institutional review board committee (July 2014; No 201407 S14P). All women provided their written informed consent and were self-reported as Caucasians. Amniotic fluid from women in this cohort was used in some of our previous studies.

\section{Amniotic Fluid IL-6 Concentrations}

The IL-6 concentrations were assessed with a lateral flow immunoassay Milenia QuickLine IL-6 using the MileniaPOCScan Reader (Millenia Biotec, Giessen, Germany). The measurement range was $50-10,000 \mathrm{pg} / \mathrm{ml}$. The intra-assay and inter-assay variations were $12.1 \%$ and $15.5 \%$, respectively.

\section{Umbilical Cord Blood IL-6 Concentrations}

IL-6 levels were assessed with enzyme-linked immunosorbent assays using the Human IL-6 Quantikine kit (R\&D Systems, Minneapolis, $\mathrm{MN})$. The sensitivity of the test was less than $0.70 \mathrm{pg} / \mathrm{ml}$, and the inter-assay and intra-assay coefficients were less than $10 \%$.

\section{Detection of Ureaplasma Species, Mycoplasma hominis, and Chlamydia trachomatis in Amniotic Fluid}

DNA was isolated from the amniotic fluid with the QIAamp DNA Mini Kit (QIAGEN, Hilden, Germany) according to the manufacturer's instructions (using the protocol for the isolation of bacterial DNA from biological fluids). Real-time PCR was performed with a Rotor-Gene 6000 instrument (QIAGEN) using a commercial AmpliSens C. trachomatis/Ureaplasma/M. hominis-FRT kit (Federal State Institution of Science, Central Research Institute of Epidemiology, Moscow, Russia) to detect DNA from Ureaplasma species, $M$. hominis, and C. trachomatis in a single PCR tube. As a control, we included a PCR run for beta-actin, a housekeeping gene to control for the presence of inhibitors of the PCR. The level of Ureaplasma species' DNA in copies/ml was determined with an absolute quantification technique employing an external calibration curve. Plasmid DNA (pCR4, Invitrogen, Carlsbad, CA) was used to prepare the calibration curve $(16,17)$.

\section{Non-Cultivation Detection of Other Bacteria in the Amniotic Fluid}

Bacterial DNA was identified by PCR targeting the 16S rRNA gene with the following primers: 5'-CCAGACTCCTACGGGAGGCAG-3' (V3 region) and 5'-ACATTTCACAACAC-GAGCTGACGA-3' (V6 region) $(18,19)$. Each individual reaction contained $3 \mu \mathrm{l}$ of target DNA, $500 \mathrm{nM}$ of forward and reverse primers, and Q5 High Fidelity DNA polymerase (NEB, Hitchin, UK) in a total volume of $25 \mu$ l. The amplification was performed in a 2720 Thermal Cycler (Applied Biosystems, Foster City, CA). The products were visualized on an agarose gel. Positive reactions yielded products of $950 \mathrm{bp}$, which were subsequently analyzed by sequencing. The $16 \mathrm{~S}$ PCR products were purified and used in sequencing PCR reactions utilizing the above primers and the BigDye Terminator kit, version 3.1 (Applied Biosystems). The bacteria were then typed by aligning the sequences obtained in BLAST and SepsiTest BLAST.

\section{Aerobic and Anaerobic Cultivations of the Amniotic Fluid}

Amniotic fluid was cultivated using these media: Columbia agar with sheep blood, Gardnerella vaginalis selective medium, MacConkey agar, Neisseria-selective medium (modified Thayer-Martin medium), Sabouraud agar, and Schaedler anaerobe agar. The plates were cultivated for 6 days, and checked daily. Species identification was provided by matrix-assisted laser desorption (MALDI)/ionization time-of-flight mass spectrometry using MALDI Biotyper software (Bruker Daltonics, Bremen, Germany; MALDI Biotyper). 


\section{Definition of IAI}

IAI in PPROM pregnancies was defined as amniotic fluid bedside IL-6 concentrations of $745 \mathrm{pg} / \mathrm{ml}$ and higher $(20,21)$.

\section{Diagnosis of MIAC}

MIAC was confirmed based on a positive PCR analysis result for Ureaplasma species, M. hominis, or C. trachomatis and/or positivity for the 16S rRNA gene and/or positive aerobic and anaerobic cultivation results from the amniotic fluid.

\section{Diagnosis of HCA}

The degree of polymorphonuclear leukocyte infiltration was assessed separately in the free membranes (amnion and chorion-decidua), in the chorionic plate, and in the umbilical cord according to the criteria given by Salafia et al. (22). Diagnosis of HCA was based on the presence of inflammatory changes in the chorion-decidua (grades 3 and 4), chorionic plate (grades 3 and 4), umbilical cord (grades 14 ), and/or amnion (grades 1-4) (22).

\section{Diagnosis of FIRS}

The presence of FIRS was diagnosed by two approaches based on: (i) the presence of umbilical cord blood IL- 6 concentrations higher than $11.0 \mathrm{pg} / \mathrm{ml} \mathrm{(8)}$ and (ii) the histopathology of the placenta characterized by the presence of funisitis (grades 1-4 in umbilical cord) and/or chorionic plate vasculitis (grade 4 in chorionic plate) (23).

\section{Diagnosis of Severe Neonatal Morbidity}

Maternal and perinatal medical records were reviewed by two investigators (M.K. and I.M.). Data regarding short-term neonatal morbidity were reviewed for all newborns. "Compound neonatal morbidity" was defined in this study as follows: the need for intubation, and/or the need for nasal continuous positive airway pressure, and/or respiratory distress syndrome (defined by the presence of two or more of the following criteria: evidence of respiratory compromise, a persistent oxygen requirement for more than $24 \mathrm{~h}$, administration of exogenous surfactant, and radiographic evidence of hyaline membrane disease), and/or transient tachypnea of newborns (defined as any oxygen supplement requirement during the first $6 \mathrm{~h}$ that does not increase during the subsequent $18 \mathrm{~h}$ as clinical conditions improve within $3-6 \mathrm{~h}$ and chest radiographs either normal or indicating reduced translucency, infiltrates, and hyperinsufflation of the lungs), and/or bronchopulmonary dysplasia (defined as infant oxygen requirement at 28 days of life), and/or pneumonia (diagnosed by abnormal findings on chest X-rays), and/ or retinopathy of prematurity (identified using retinoscopy), and/or intraventricular hemorrhage (the diagnosis of which was made using cranial ultrasound examinations according to the procedure of Papile et al. (24)), and/or necrotizing enterocolitis (defined as radiologic findings of either intramural gas or free intra-abdominal gas), and/or early- (during the first $72 \mathrm{~h}$ of life) or late-onset (between the ages of 4 and 120 days) sepsis (either proven by bacterial culture or clinically highly suspected sepsis), and/or neonatal death before hospital discharge.

\section{Statistical Analysis}

The demographic and clinical characteristics were compared using the nonparametric Kruskal-Wallis test and presented as medians (interquartile range (IQR)) for continuous variables. Categorical variables were compared using the $\chi^{2}$-test and were presented as numbers (\%). The normality of the data was tested using the D'Agostino and Pearson omnibus normality test. Because the umbilical cord blood concentrations of IL-6 were not normally distributed, the nonparametric Mann-Whitney U-test or KruskalWallis test was used for the analyses, as appropriate. The rates of short-term neonatal morbidity were compared using the Fisher's exact test or the Cochran-Armitage test for trends as appropriate. The results were adjusted for potential confounders using the partial Spearman correlation. Differences were considered statistically significant at $P<0.05$. All $P$ values were from two-sided tests, and all statistical analyses were performed using SPSS 19.0 for Mac OS X (SPSS, Chicago, IL) and with GraphPad Prism $6.0 \mathrm{~h}$ for Mac OS X (GraphPad Software, La Jolla, CA).

\section{RESULTS}

\section{Demographic and Clinical Characteristics of the Study Population}

In total, 171 women with PPROM at gestational ages $34+0$ and $36+6$ were included in the study. Twelve women were excluded because of gestational diabetes mellitus $(n=10)$, diabetes mellitus $(n=1)$, and late-onset preeclampsia $(n=1)$. The remaining 159 women were included in the analysis.

MIAC and IAI were both present in 9\% (15/159) of women, IAI alone was found in 5\% (8/159), MIAC alone was identified in $19 \%(29 / 159)$, and $67 \%$ (107/159) of women had neither MIAC nor IAI. The maternal and neonatal characteristics of the study group according to the presence of MIAC and/or IAI are shown in Table 1. All microbial findings from amniotic fluid are shown in Table 2.

The presence of FIRS was detected in 23\% (37/159) and $33 \%(52 / 159)$ of women based on the umbilical cord blood IL-6 concentrations and histopathological findings of the placentas, respectively. Of the subjects, $13 \%$ (20/159) of women fulfilled both criteria, 11\% (17/159) of women were diagnosed only based on umbilical cord blood IL-6 concentrations, and 20\% (32/159) were diagnosed only based on the histopathological findings.

\section{Umbilical Cord Blood IL-6 in Relation to the Presence of MIAC and/or IAI}

A difference was found in umbilical cord blood IL-6 concentrations among the subgroups of women with and without MIAC and/or IAI in crude analysis (with both MIAC and IAI: median $27.1 \mathrm{pg} / \mathrm{ml}$, IQR $7.1-52.3$ vs. with IAI alone: median $3.8 \mathrm{pg} / \mathrm{ml}$, IQR 2.4-35.8 vs. with MIAC alone: median $3.8 \mathrm{pg} / \mathrm{ml}$, IQR 2.6-9.6 vs. without both IAI and MIAC: median $4.5 \mathrm{pg} / \mathrm{ml}$, IQR 2.3-7.5; $P=0.003$; Figure 1) as well as after the adjustment for the gestational age at delivery, administration of corticosteroids and antibiotics, prepregnancy $\mathrm{BMI}$, and smoking $(P<0.0001)$. A weak correlation was found between microbial load of Ureaplasma species (DNA copies $/ \mathrm{ml}$ ) in the amniotic fluid and amniotic fluid IL-6 concentrations (rho $=0.37 ; P=0.04)$, but not with umbilical cord blood IL-6 concentrations (rho $=0.29$; $P=0.12)$.

\section{The Presence of FIRS in Pregnancies With and Without MIAC and/or IAI}

FIRS defined on the basis of umbilical cord blood IL-6 concentrations was found in $73 \%(11 / 15)$ of women with both IAI and MIAC, in 38\% (3/8) with IAI alone, in $21 \%(6 / 29)$ with MIAC alone, and in $16 \%(17 / 107)$ without both IAI and MIAC. A difference in the rates of FIRS among these subgroups was observed in the crude and adjusted analyses (both $P<0.0001$ ). When defined based on the 
Table 1. Demographic and clinical characteristics of the women with PPROM between the gestational ages of 34 and 37 weeks stratified according to the presence of IAI and/or MIAC

\begin{tabular}{|c|c|c|c|c|c|}
\hline Characteristic & $\begin{array}{l}\text { With MIAC and IAI } \\
\qquad(n=15)\end{array}$ & $\begin{array}{l}\text { With IAI alone } \\
\qquad(n=8)\end{array}$ & $\begin{array}{l}\text { With MIAC alone } \\
\quad(n=29)\end{array}$ & $\begin{array}{l}\text { Without MIAC and IAI } \\
\qquad(n=107)\end{array}$ & $P$ value \\
\hline Maternal age (years, median (IQR)) & $29(25-30)$ & $30(26-34)$ & $30(25-33)$ & $30(28-34)$ & 0.58 \\
\hline Primiparous (number (\%)) & $6(40 \%)$ & $6(75 \%)$ & $12(41 \%)$ & $68(64 \%)$ & 0.06 \\
\hline Prepregnancy BMI $\left(\mathrm{kg} / \mathrm{m}^{2}\right.$, median (IQR)) & $22.5(19.5-26.7)$ & $21.8(19.8-25.2)$ & $21.9(19.1-24.4)$ & $23.3(21.5-27.3)$ & 0.03 \\
\hline Smoking (number (\%)) & $5(33 \%)$ & $0(0 \%)$ & 7 (24\%) & $6(6 \%)$ & 0.009 \\
\hline $\begin{array}{l}\text { Gestational age at admission (weeks, median } \\
\text { (IQR)) }\end{array}$ & $35+2(34+4-36+2)$ & $\begin{array}{c}35+1(34+6-36 \\
+3)\end{array}$ & $35+3(34+4-36+3)$ & $35+3(34+6-36+2)$ & 0.99 \\
\hline $\begin{array}{l}\text { Gestational age at delivery (weeks, median } \\
\text { (IQR)) }\end{array}$ & $35+3(34+5-36+2)$ & $\begin{array}{c}35+5(35+0-36 \\
+2)\end{array}$ & $35+4(35+0-36+4)$ & $35+5(35+0-36+3)$ & 0.93 \\
\hline $\begin{array}{l}\text { Interval from PPROM to amniocentesis ( } h \text {, } \\
\text { median (IQR)) }\end{array}$ & $8(4-14)$ & $6(2-19)$ & $4(3-7)$ & $4(3-8)$ & 0.09 \\
\hline $\begin{array}{l}\text { Latency from amniocentesis to delivery ( } h \text {, } \\
\text { median (IQR)) }\end{array}$ & $17(8-29)$ & $29(4-47)$ & $31(10-62)$ & $23(11-48)$ & 0.44 \\
\hline WBC count at admission $\left(\times 10^{9} \mathrm{I}\right.$, median (IQR)) & $13.2(10.0-15.8)$ & $16.5(14.7-21.9)$ & $11.8(9.2-13.9)$ & $11.4(9.7-13.2)$ & 0.03 \\
\hline Administration of antibiotics (number (\%)) & $15(100 \%)$ & $8(100 \%)$ & $28(97 \%)$ & $104(97 \%)$ & 0.87 \\
\hline Administration of corticosteroids (number (\%)) & $5(33 \%)$ & $2(25 \%)$ & $9(31 \%)$ & $30(28 \%)$ & 0.96 \\
\hline Spontaneous vaginal delivery (number (\%)) & $14(93 \%)$ & $6(75 \%)$ & $23(79 \%)$ & $86(80 \%)$ & 0.62 \\
\hline Forceps delivery (number (\%)) & $0(0 \%)$ & $0(0 \%)$ & $0(0 \%)$ & $2(2 \%)$ & 0.81 \\
\hline Cesarean delivery (number (\%)) & $1(7 \%)$ & $2(25 \%)$ & $6(21 \%)$ & $17(16 \%)$ & 0.60 \\
\hline Birth weight (g, median (IQR)) & $2,680(2,170-2,840)$ & $\begin{array}{c}2,575(2,213- \\
2,855)\end{array}$ & $2,520(2,330-2,835)$ & $2,580(2,290-2,790)$ & 0.99 \\
\hline Histological chorioamnionitis (number (\%)) & $11(73 \%)$ & $5(63 \%)$ & $16(55 \%)$ & $42(39 \%)$ & 0.04 \\
\hline
\end{tabular}

Table 2. The bacterial species identified in the amniotic fluid of women with PPROM between gestational ages 34 and 37 weeks

\begin{tabular}{ll}
\hline Women with MIAC and IAI $(n=15)$ & Women with MIAC alone $(n=29)$ \\
\hline Ureaplasma species $9 \times$ & Ureaplasma species $15 \times$ \\
Ureaplasma species+Mycoplasma hominis $2 \times$ & Mycoplasma hominis $3 \times$ \\
Haemophilus influenza $1 \times$ & Propionibacterium acnes $2 \times$ \\
Ureaplasma species+Sneathia sanguinegens $1 \times$ & Streptococcus intermedius $1 \times$ \\
Ureaplasma species+Enterococcus faecium $1 \times$ & Staphylococcus warneri $1 \times$ \\
Peptococcus species+Propionibacterium species+Bacteroides species $1 \times$ & Enterococcus faecium $1 \times$ \\
& Chlamydia trachomatis $1 \times$ \\
& Lactobacillus gasseri $1 \times$ \\
& Lactobacillus gasseri+Bifidobacterium breve $1 \times$ \\
& Ureaplasma species+Chlamydia trachomatis $1 \times$ \\
& Streptococcus salivarius+Enterococcus faecalis + Lactobacillus crispatus $1 \times$ \\
& Ureaplasma species+Chlamydia trachomatis + Lactobacillus crispatus $1 \times$ \\
\hline
\end{tabular}

IAI, intra-amniotic inflammation; MIAC, microbial invasion of the amniotic cavity; PPROM, preterm prelabor rupture of membranes. 


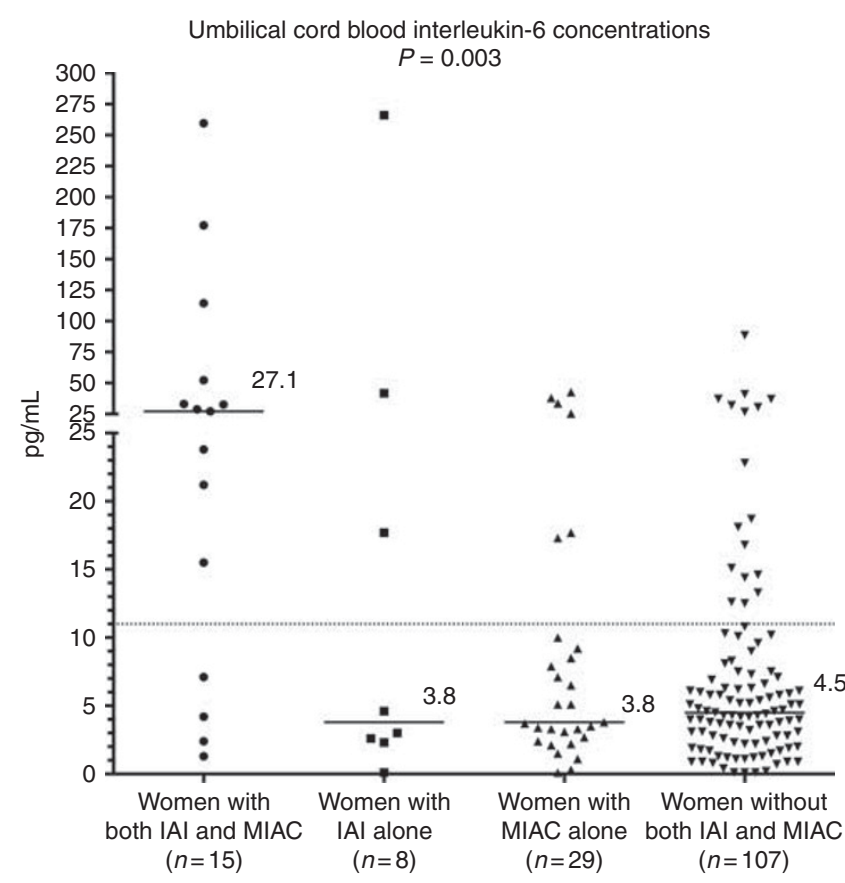

Figure 1. Umbilical cord blood IL-6 concentrations in preterm prelabor rupture of membranes in presence of IAI and/or MIAC. Horizontal bars represent medians. The dotted line indicates the cutoff defining FIRS based on umbilical cord blood IL- 6 concentrations. FIRS, fetal inflammatory response syndrome; IAI, intra-amniotic inflammation; IL, interleukin; MIAC, microbial invasion of the amniotic cavity. histopathological criteria, FIRS was identified in 67\% (10/15) of women with both IAI and MIAC, in 38\% (3/8) of women with IAI alone, in 46\% (16/29) with MIAC alone, and in $24 \%$ (26/107) without both IAI and MIAC. A difference in the rates of FIRS among these subgroups was identified in the crude $(P=0.0005)$ and adjusted $(P=0.002)$ analyses.

\section{The Presence of FIRS and the Selected Aspects of Short-Term Neonatal Morbidity}

Women with FIRS had higher rates of early-onset sepsis $(P=0.01$; Table 3$)$ and intraventricular hemorrhage grades I and II $(P=0.04$; Table 3$)$ when FIRS was characterized based on the umbilical cord blood IL-6 concentrations and the histopathological findings, respectively. The differences remained significant after the adjustment for the gestational age at delivery, administration of corticosteroids and antibiotics, pre-pregnancy BMI, and smoking $(P=0.001$ and $P=0.02$ ). No differences in the other aspects of short-term neonatal morbidity were identified.

Two cases of early-onset sepsis were clinically suspected and one was confirmed by a positive bacterial culture. All the cases had amniotic fluid IL-6 concentrations $10,000 \mathrm{pg} / \mathrm{ml}$. Two cases were from pregnancies with the presence of Ureaplasma species in amniotic fluid and one case was from pregnancy with the presence of Haemophilus influenzae in amniotic

Table 3. Selected aspects of short-term neonatal morbidity with respect to the presence or absence of fetal inflammatory response (based on umbilical cord blood IL-6 cutoff value $11 \mathrm{pg} / \mathrm{ml}$ and histopathology of the placenta and fetal membranes)

\begin{tabular}{|c|c|c|c|c|c|c|c|c|}
\hline & \multicolumn{2}{|c|}{ The presence of FIRS } & \multicolumn{2}{|c|}{ The absence of FIRS } & \multirow[t]{2}{*}{$P$ value ${ }^{1}$} & \multirow[t]{2}{*}{$P$ value $^{2}$} & \multirow[t]{2}{*}{$P$ value $^{3}$} & \multirow[t]{2}{*}{$P$ value } \\
\hline & IL-6 $(n=37)$ & Histology $(n=52)$ & IL-6 $(n=122)$ & Histology $(n=107)$ & & & & \\
\hline Transient tachypnea of newborns & $3(8 \%)$ & $2(4 \%)$ & $4(3 \%)$ & $5(5 \%)$ & 0.36 & 0.14 & 1.00 & 0.88 \\
\hline Respiratory disorders & $4(11 \%)$ & $2(4 \%)$ & $9(7 \%)$ & $11(10 \%)$ & 0.50 & 0.38 & 0.22 & 0.17 \\
\hline Need for intubation & $0(0 \%)$ & $1(2 \%)$ & $2(2 \%)$ & $1(1 \%)$ & 1.00 & 0.47 & 0.55 & 0.54 \\
\hline Early-onset sepsis & $3(8 \%)$ & $2(4 \%)$ & $0(0 \%)$ & $1(1 \%)$ & 0.01 & 0.001 & 1.00 & 0.93 \\
\hline Late-onset sepsis & $0(0 \%)$ & $0(2 \%)$ & $2(2 \%)$ & $2(2 \%)$ & 1.00 & 0.36 & 1.00 & 0.27 \\
\hline Compound neonatal morbidity & $6(16 \%)$ & $6(12 \%)$ & $13(11 \%)$ & $13(12 \%)$ & 0.39 & 0.26 & 1.00 & 0.95 \\
\hline Admission at NICU & $5(14 \%)$ & $5(10 \%)$ & $11(9 \%)$ & $11(10 \%)$ & 0.53 & 0.33 & 1.00 & 0.92 \\
\hline
\end{tabular}

FIRS, fetal inflammatory response syndrome; IL, interleukin; nCPAP, nasal continuous positive airway pressure; PPROM, preterm prelabor rupture of membranes; NICU, newborns' intensive care unit.

Respiratory disorders were defined as a respiratory distress syndrome or transient tachypnea of newborns.

Compound neonatal morbidity was defined as a need for intubation and/or a need for nasal continuous positive airway pressure and/or respiratory distress syndrome and/or transient tachypnea of newborns and/or pneumonia and/or bronchopulmonary dysplasia and/or retinopathy of prematurity and/or intraventricular hemorrhage and/or necrotizing enterocolitis and/or early-onset sepsis and/or late-onset sepsis and/or neonatal death before hospital discharge.

Intraventricular hemorrhage grades III and IV, bronchopulmonary dysplasia, retinopathy of prematurity, necrotizing enterocolitis, and neonatal death before hospital discharge were not considered in the analysis because of no occurrence in the cohort.

Categorical variables were compared using Fisher's exact test. Spearman partial correlation was used to adjust the data for the gestational age at delivery. Statistically significant results are marked in bold.

$P$ value 1 , the comparison between women with and without FIRS defined based on umbilical cord blood IL-6.

$P$ value ${ }^{2}$, the comparison between women with and without FIRS defined based on umbilical cord blood IL-6 adjusted for the gestational age at delivery, administration of corticosteroids and antibiotics, pre-pregnancy BMl, and smoking.

$P$ value $^{3}$, the comparison between women with and without FIRS defined based on the histopathology of the placenta and fetal membranes.

$P$ value ${ }^{4}$, the comparison between women with and without FIRS defined based on the histopathology of the placenta and fetal membranes adjusted for the gestational age

at delivery, administration of corticosteroids and antibiotics, pre-pregnancy BMI, and smoking. 
fluid. The newborn from this pregnancy developed cultureproven early-onset sepsis (H. influenzae).

\section{Selected Aspects of Short-Term Neonatal Morbidity According to the Gestational Age of Delivery}

A decreasing trend of compound neonatal morbidity (19\% $(9 / 48)$ in gestational ages $34+0-34+6,15 \%(8 / 53)$ in gestational ages $35+0-35+6$, and $3 \%(2 / 58)$ in gestational ages $36+0-36+6 ; P=0.01$ ) was found among women with PPROM between 34 and 37 weeks of gestation. No differences in the other aspects of short-term neonatal morbidity analyzed were identified.

\section{Discussion}

Principal findings of this study. First, the presence of both MIAC and IAI was associated with the highest umbilical cord blood IL-6 concentrations and the highest rate of FIRS. Second, no correlation between the microbial loads of Ureaplasma species in amniotic fluid and umbilical cord blood IL-6 concentrations was identified. Third, the presence of FIRS, defined by umbilical cord blood IL-6 concentrations, was related to a higher rate of early-onset sepsis. Fourth, the presence of FIRS, defined by histopathology of the placenta, was associated with a higher rate of intraventricular hemorrhage grades I and II. Finally, compound neonatal morbidity decreased with advanced gestational age.

Meaning of the study. An inflammatory response is elicited in the fetus and newborns through the innate immune system by the activation of pattern recognition receptors, which are able to recognize specific motifs on the surface of microorganism or endogenous molecules called alarmins (2527). This means that the fetal inflammatory response might be triggered by either IAI with MIAC or IAI alone (sterile IAI). In spite of the fact that both types of IAI lead to a fetal inflammatory response, it is still not clear whether the concurrent presence of MIAC in women with PPROM complicated by IAI might result in an even stronger fetal inflammatory response. Lee $e t$ al. described a stronger intensity of the fetal inflammatory response, measured by C-reactive protein levels, when both MIAC and IAI were present (28). Conversely, our group has not identified any difference in the intensity of the fetal inflammatory response based on IL-6 levels (29). The occurrence of IAI alone in PPROM between 34 and 37 weeks of gestation was relatively infrequent. Therefore, the small sample size of the subgroup of women with IAI alone prevents us from making a definitive conclusion regarding the intensity of fetal inflammatory response for the two types of IAI.

Ureaplasma species, considered low virulent bacteria, have been reported as the most common microorganism identified in amniotic fluid in women with PPROM $(30,31)$. An inflammatory response to Ureaplasma species in intraamniotic maternal and fetal compartments has been shown to be dose-dependent $(4,17,32-34)$. Surprisingly, we did not find any correlation between the microbial load of Ureaplasma species and umbilical cord blood IL-6 concentrations in this study. This result appears to be clinically relevant since the evaluation of the microbial load of Ureaplasma species in PPROM between 34 and 37 weeks of gestation does not provide clinically important information such as the prediction of the intensity of the fetal inflammatory response.

FIRS is a consequence of the systemic activation of the fetal innate immune system. FIRS was originally described by Gomez et al. in 1998 as umbilical cord blood IL-6 concentration higher than $11 \mathrm{pg} / \mathrm{ml}$ in pregnancies complicated with PPROM and spontaneous preterm labor with intact membranes (8). Four years later, the presence of funisitis and/or chorionic plate vasculitis was identified by Pacora et al. as a histopathological counterpart of FIRS (23). In 2013, Lee et al. suggested using the term FIRS type I for the above-mentioned types of FIRS, and they introduced a new type of FIRS, defined as FIRS type II, for fetuses with inflammatory response with evidence of maternal antifetal rejection (35). In this study, we focused our attention only on FIRS type I, and particularly on the differences in short-term neonatal outcome when FIRS type I is defined based on umbilical cord blood IL-6 levels and on the histopathology of the placenta. FIRS defined as having umbilical cord blood IL- 6 concentrations greater than $11 \mathrm{pg} / \mathrm{ml}$ was found to be correlated with a higher rate of early-onset sepsis. This finding is in line with the results from other studies as well as our own performed in women with PPROM $(8,10,29)$. However, when FIRS was determined based on the histopathology of the placenta, the present study revealed higher rates of intraventricular hemorrhage grades I and II, but not of early-onset sepsis. However, this finding is interesting as it is in line with the theory that the presence of histologic chorioamnionitis contributes to the risk of brain injury in PPROM $(36,37)$. It is not our intention to overemphasize these findings, given the low incidence of early-onset sepsis $(2 \% ; 3 / 159)$ and intraventricular hemorrhage grades I and II $(3 \% ; 5 / 159)$ observed in women with PPROM between 34-37 weeks of gestation in our study; nonetheless, the association of differently defined FIRS type I and the neonatal outcomes based on these definitions should be evaluated further in larger studies.

Gestational age at delivery has been shown to be a main determinant of short-term neonatal morbidity, even in PPROM between 34 and 37 weeks of gestation $(12,38)$. In this study, we confirmed that compound neonatal morbidity decreases with advanced gestational age even in this very-tight time frame covering just 3 gestational weeks. This finding might further support the results from a recent study showing that the expectant management of PPROM between 34 and 37 weeks of gestation can improve neonatal outcomes (15).

The main strength of this study is the relatively large cohort of women with PPROM between the gestational ages of 34 and 37 weeks from a single tertiary referral center. Second, the unique aspect of this study is the fact that it contains data regarding intra-amniotic, fetal, and placental environments combined with short-term neonatal outcomes. Third, MIAC was defined based on the combination of non-cultivation (nonspecific PCR for 16S rRNA and specific PCR for 


\section{Articles | Musilova et al.}

Ureaplasma species, $M$. hominis, and C. trachomatis) and cultivation approaches. Fourth, the IL-6 level was selected as a marker of the intensity of the fetal inflammatory response as its umbilical cord blood concentrations are not affected by the gestational age of the sample (39).

There are some limitations in this study. First, the umbilical cord blood samples were obtained non-invasively by venipuncture of the umbilical cord after delivery of the newborn. This approach prevented us from sampling cord blood before the administration of the corticosteroids and antibiotics. Therefore, we cannot rule out the potential effect of these agents on umbilical cord blood IL-6 concentrations. To address this potentially confounding factor, the results were adjusted for the administration of antibiotics and corticosteroids. Second, this study was limited by the relatively small sample sizes of the subgroups with both IAI and MIAC and women with IAI alone, which increases the potential for a type II error.

In summary, the presence of both MIAC and IAI was associated with a higher fetal inflammatory response and a higher rate of FIRS in women with PPROM between the gestational ages of 34 and 37 weeks. Different aspects of shortterm neonatal morbidity were related to FIRS when defined by umbilical cord blood IL-6 concentrations and the histopathology of the placenta.

\section{STATEMENT OF FINANCIAL SUPPORT}

This work was supported by the Faculty Hospital in Hradec Kralove (longterm organization development plan) and by the Charles University in Prague, Faculty of Medicine in Hradec Kralove, Czech Republic, project "PROGRES Q40".

Disclosure: The authors declare no conflict of interest.

\section{REFERENCES}

1. Mercer BM. Preterm premature rupture of the membranes. Obstet Gynecol 2003;101:178-93.

2. Mercer BM. Preterm premature rupture of the membranes: diagnosis and management. Clin Perinatol 2004;31:765-82.

3. Caughey AB, Robinson JN, Norwitz ER. Contemporary diagnosis and management of preterm premature rupture of membranes. Rev Obstet Gynecol 2008;1:11-22.

4. Musilova I, Kutova R, Pliskova L, et al. Intraamniotic inflammation in women with preterm prelabor rupture of membranes. PLoS ONE 2015;10:e0133929.

5. DiGiulio DB, Romero R, Kusanovic JP, et al. Prevalence and diversity of microbes in the amniotic fluid, the fetal inflammatory response, and pregnancy outcome in women with preterm pre-labor rupture of membranes. Am J Reprod Immunol 2010;64:38-57.

6. Romero R, Miranda J, Chaemsaithong P, et al. Sterile and microbialassociated intra-amniotic inflammation in preterm prelabor rupture of membranes. J Matern Fetal Neonatal Med 2015;28:1394-409.

7. Kim CJ, Romero R, Chaemsaithong P, Chaiyasit N, Yoon BH, Kim YM. Acute chorioamnionitis and funisitis: definition, pathologic features, and clinical significance. Am J Obstet Gynecol 2015;213:S29-52.

8. Gomez R, Romero R, Ghezzi F, Yoon BH, Mazor M, Berry SM. The fetal inflammatory response syndrome. Am J Obstet Gynecol 1998;179: 194-202.

9. Gotsch F, Romero R, Kusanovic JP, et al. The fetal inflammatory response syndrome. Clin Obstet Gynecol 2007;50:652-83.
10. Hofer N, Kothari R, Morris N, Muller W, Resch B. The fetal inflammatory response syndrome is a risk factor for morbidity in preterm neonates. Am J Obstet Gynecol 2013;209:542 e1-11.

11. Romero R, Savasan ZA, Chaiworapongsa T, et al. Hematologic profile of the fetus with systemic inflammatory response syndrome. J Perinat Med 2011;40:19-32.

12. Kacerovsky M, Musilova I, Andrys C, et al. Prelabor rupture of membranes between 34 and 37 weeks: the intraamniotic inflammatory response and neonatal outcomes. Am J Obstet Gynecol 2014;210: 325 e1-10.

13. van der Ham DP, van der Heyden JL, Opmeer BC, et al. Management of late-preterm premature rupture of membranes: the PPROMEXIL-2 trial. Am J Obstet Gynecol 2012;207:276 e1-10.

14. van der Ham DP, Vijgen SM, Nijhuis JG, et al. Induction of labor versus expectant management in women with preterm prelabor rupture of membranes between 34 and 37 weeks: a randomized controlled trial. PLoS Med 2012;9:e1001208.

15. Morris JM, Roberts CL, Bowen JR, et al. Immediate delivery compared with expectant management after preterm pre-labour rupture of the membranes close to term (PPROMT trial): a randomised controlled trial. Lancet 2016;387:444-52.

16. Tambor V, Kacerovsky M, Andrys C, et al. Amniotic fluid cathelicidin in PPROM pregnancies: from proteomic discovery to assessing its potential in inflammatory complications diagnosis. PLoS ONE 2012;7: e41164.

17. Kacerovsky M, Pliskova L, Bolehovska R, et al. The microbial load with genital mycoplasmas correlates with the degree of histologic chorioamnionitis in preterm PROM. Am J Obstet Gynecol 2011;205: 213 e1-7.

18. Fouhy F, Deane J, Rea MC, et al. The effects of freezing on faecal microbiota as determined using MiSeq sequencing and culture-based investigations. PLoS ONE 2015;10:e0119355.

19. Greisen K, Loeffelholz M, Purohit A, Leong D. PCR primers and probes for the 16S rRNA gene of most species of pathogenic bacteria, including bacteria found in cerebrospinal fluid. J Clin Microbiol 1994;32:335-51.

20. Chaemsaithong P, Romero R, Korzeniewski SJ, et al. A rapid interleukin6 bedside test for the identification of intra-amniotic inflammation in preterm labor with intact membranes. J Matern Fetal Neonatal Med 2015;29:1-11.

21. Chaemsaithong P, Romero R, Korzeniewski SJ, et al. A point of care test for interleukin-6 in amniotic fluid in preterm prelabor rupture of membranes: a step toward the early treatment of acute intra-amniotic inflammation/infection. J Matern Fetal Neonatal Med 2016;29:360-7.

22. Salafia CM, Weigl C, Silberman L. The prevalence and distribution of acute placental inflammation in uncomplicated term pregnancies. Obstet Gynecol 1989;73:383-9.

23. Pacora $\mathrm{P}$, Chaiworapongsa T, Maymon E, et al. Funisitis and chorionic vasculitis: the histological counterpart of the fetal inflammatory response syndrome. J Matern Fetal Neonatal Med 2002;11:18-25.

24. Papile LA, Burstein J, Burstein R, Koffler H. Incidence and evolution of subependymal and intraventricular hemorrhage: a study of infants with birth weights less than 1,500 gm. J Pediatr 1978;92:529-34.

25. Sugitharini V, Pavani K, Prema A, Berla Thangam E. TLR-mediated inflammatory response to neonatal pathogens and co-infection in neonatal immune cells. Cytokine 2014;69:211-7.

26. Koga K, Izumi G, Mor G, Fujii T, Osuga Y. Toll-like receptors at the maternal-fetal interface in normal pregnancy and pregnancy complications. Am J Reprod Immunol 2014;72:192-205.

27. Medzhitov R, Janeway CA Jr. Decoding the patterns of self and nonself by the innate immune system. Science 2002;296:298-300.

28. Lee SE, Romero R, Jung H, Park CW, Park JS, Yoon BH. The intensity of the fetal inflammatory response in intraamniotic inflammation with and without microbial invasion of the amniotic cavity. Am J Obstet Gynecol 2007;197:291.e1-6.

29. Musilova I, Andrys C, Drahosova M, et al. Intraamniotic inflammation and umbilical cord blood interleukin- 6 concentrations in pregnancies 
complicated by preterm prelabor rupture of membranes. J Matern Fetal Neonatal Med 2017;30:900-10.

30. Kacerovsky M, Celec P, Vlkova B, et al. Amniotic fluid protein profiles of intraamniotic inflammatory response to Ureaplasma spp. and other bacteria. PLoS ONE 2013;8:e60399.

31. Oh KJ, Lee KA, Sohn YK, et al. Intraamniotic infection with genital mycoplasmas exhibits a more intense inflammatory response than intraamniotic infection with other microorganisms in patients with preterm premature rupture of membranes. Am J Obstet Gynecol 2010;203:211 e1-8.

32. Jacobsson B, Aaltonen R, Rantakokko-Jalava K, Morken NH, Alanen A. Quantification of Ureaplasma urealyticum DNA in the amniotic fluid from patients in PTL and PPROM and its relation to inflammatory cytokine levels. Acta Obstet Gynecol Scand 2009;88:63-70.

33. Kasper DC, Mechtler TP, Reischer GH, et al. The bacterial load of Ureaplasma parvum in amniotic fluid is correlated with an increased intrauterine inflammatory response. Diagn Microbiol Infect Dis 2010;67:117-21.

34. Kacerovsky M, Pliskova L, Bolehovska R, et al. The impact of the microbial load of genital mycoplasmas and gestational age on the intensity of intraamniotic inflammation. Am J Obstet Gynecol 2012;206:342 e1-8.
35. Lee J, Romero R, Chaiworapongsa T, et al. Characterization of the fetal blood transcriptome and proteome in maternal anti-fetal rejection: evidence of a distinct and novel type of human fetal systemic inflammatory response. Am J Reprod Immunol 2013;70:265-84.

36. Lu HY, Zhang Q, Wang QX, Lu JY. Contribution of histologic chorioamnionitis and fetal inflammatory response syndrome to increased risk of brain injury in infants with preterm premature rupture of membranes. Pediatr Neurol 2016;61:94-8 e91.

37. Lu H, Wang Q, Lu J, Zhang Q, Kumar P. Risk factors for intraventricular hemorrhage in preterm infants born at 34 weeks of gestation or less following preterm premature rupture of membranes. J Stroke Cerebrovasc Dis 2016;25:807-12.

38. Rodriguez-Trujillo A, Cobo T, Vives I, et al. Gestational age is more important for short-term neonatal outcome than microbial invasion of the amniotic cavity or intra-amniotic inflammation in preterm prelabor rupture of membranes. Acta Obstet Gynecol Scand 2016;95: 926-33.

39. Matoba N, Yu Y, Mestan K, et al. Differential patterns of 27 cord blood immune biomarkers across gestational age. Pediatrics 2009;123: $1320-8$. 